\title{
Flow Patterns and Transport in Rayleigh Surface Acoustic Wave Streaming: Combined Finite Element Method and Raytracing Numerics versus Experiments
}

\author{
Thomas Frommelt, Daniel Gogel, Marcin Kostur, Peter Talkner, Peter Hänggi, \\ and Achim Wixforth, Member, IEEE
}

\begin{abstract}
This work presents an approach for determining the streaming patterns that are generated by Rayleigh surface acoustic waves in arbitrary 3 -D geometries by finite element method (FEM) simulations. An efficient raytracing algorithm is applied on the acoustic subproblem to avoid the unbearable memory demands and computational time of a conventional FEM acoustics simulation in 3-D. The acoustic streaming interaction is modeled by a body force term in the Stokes equation. In comparisons between experiments and simulated flow patterns, we demonstrate the quality of the proposed technique.
\end{abstract}

\section{INTRODUCTION}

$\mathrm{D}$ URING the recent decade, small microfluidic devices known as "labs-on-a-chip" have gained increasing practical importance mainly in the fields of microbiology and medicine where they are employed as flexible tools, e.g., for analysis, diagnostics, and research. Of central importance for most of these purposes is the controlled transport of fluids as well as of immersed objects ranging from large molecules to whole cells. In this context, surface acoustic waves (SAW) have proved to be a versatile means to actuate the pertinent small amounts of fluids in these systems [1]-[5].

The optimization as well as the reliable operation and control of these devices require a detailed understanding of the combined fluid and particle motion in small confined geometries. Although the fundamental laws governing the physics of such processes are known, their detailed quantitative understanding is severely hampered by the presence of the extremely wide ranges of the time and length scales being relevant for SAW driven flows. The flow on large length and time scales originates from the SAW excitation acting on much shorter length and time scales. In the experiments presented in this paper, the frequency is of the

Manuscript received September 5, 2007; accepted April 14, 2008. This work was supported in part by the Deutsche Forschungsgemeinschaft DFG under contracts SPP 1164, SPP 1253 and SFB 486 project B13, and in part by the German government through the Cluster of Excellence "NIM."

T. Frommelt, D. Gogel, P. Talkner, P. Hänggi, and A. Wixforth are with the Universität Augsburg, Institut für Physik, Augsburg, Germany (e-mail: thomas.frommelt@physik.uni-augsburg.de).

M. Kostur is with the University of Silisia, Institute of Physics, Katowice, Poland.

Digital Object Identifier 10.1109/TUFFC.928 order of $100 \mathrm{MHz}$ and the corresponding wavelengths on the substrate and in the fluid of the order of $10 \mu \mathrm{m}$. The transport processes, however, are characterized by time scales of at least one tenth of a second up to a few minutes and by length scales between a tenth of a millimeter up to a centimeter. A solution of the full compressible NavierStokes equations, which govern the physics of fluid motion including the propagation of acoustic waves, for a problem covering 9 orders of magnitude of time scales and 4 orders of magnitude of length scales in 3 spatial dimensions is far beyond the capability of present-day computers.

To avoid the above-mentioned difficulties, we broke down the total problem into 3 subproblems, 2 of which concern the fluid motion separated into the acoustic and the slowly streaming flow. The particle transport is assumed to be purely advective. This means that the suspended objects must be sufficiently small compared with the outer dimensions of the fluid volume and that back reactions of the objects onto the fluid motion can be ignored. For the sake of simplicity, any interactions between the particles also are ignored although, in principle, hydrodynamic and possible electric charge and dipole interactions can be taken into account.

The first fluidic module describes the propagation of sound waves within the ray approximation, which corresponds to the description of electromagnetic waves in the approximation of geometrical optics. This approximation is justified because the wavelength of the ultrasound irradiated by the SAW is short compared with the outer dimensions of the fluid volume. In the framework of the proposed modeling, each ray carries a wave that is attenuated due to viscosity and thermal conductivity of the fluid. Caused by the nonlinear, compressible Navier-Stokes equations, this attenuated wave imposes an effective force on the fluid and leads to the so-called acoustic streaming effect [6].

This effective force is used in the second module to drive the fluid on a time scale that is much larger than the period of the acoustic wave. On this large time scale, the fluid (water in our case) can be considered as incompressible. Because of the effective small streaming velocities, the nonlinear terms in the Navier-Stokes equation can be ignored, and the resulting Stokes equation can be numerically solved in the prescribed geometry. 


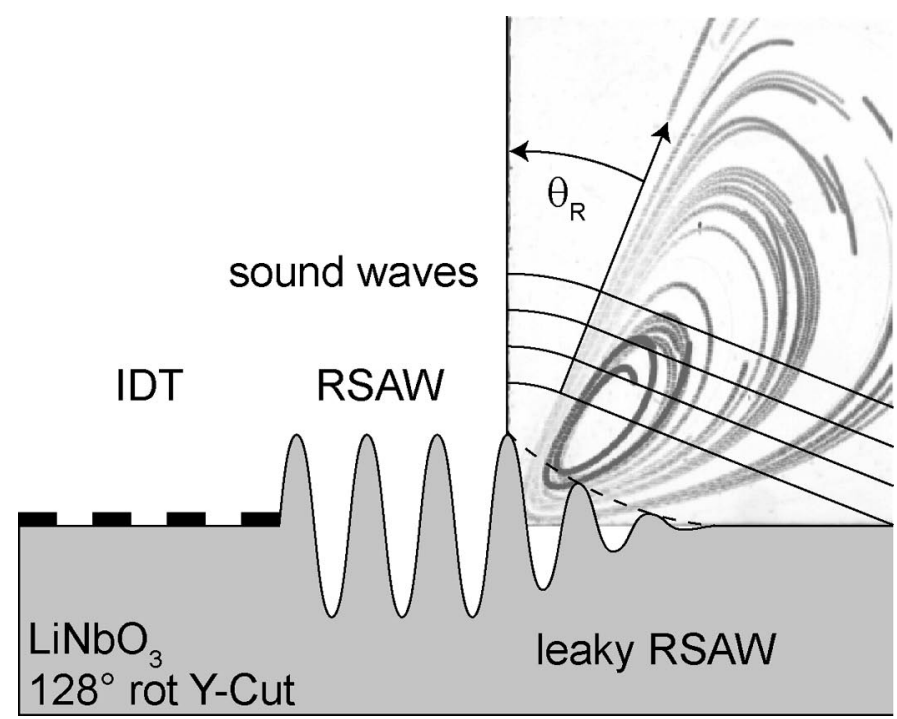

Fig. 1. Acoustic streaming: RSAWs are excited by means of an IDT. Underneath a liquid, the RSAW turns into a leaky RSAW, radiating sound waves at the Rayleigh angle $\Theta_{R}$ into the fluid. The thick gray curves indicate stream lines of the flow field driven by the RSAW.

The third module describes the advective motion of small particles in the velocity field that emerges from the second module.

\section{Rayleigh Surface Acoustic Waves}

The coupling between SAWs to the acoustic modes in a fluid is optimal for so-called Rayleigh SAW (RSAW) [7], which are elliptically polarized waves with one displacement component pointing out of the substrate plane on which the wave propagates and a second displacement component in the plane of the surface (see Fig. 1).

Pure RSAWs exist on surfaces of single crystals of lithium niobate $\left(\mathrm{LiNbO}_{3}\right)$ with a $128^{\circ}$ rotated Y-cut. This common cut provides 2 orthogonal directions with high piezoelectric coupling parallel and normal to the X crystal axis. The electromechanical coupling coefficients and the sound velocities of these 2 directions differ slightly, but the RSAW amplitudes along these propagation directions are well sufficient for the microfluidic applications presented here. Hence, these directions are commonly used and referred to in the remaining text.

The piezoelectricity of $\mathrm{LiNbO}_{3}$ allows excitation of these modes by means of radio frequency $(\mathrm{RF})$ electric fields that are applied to interdigital transducers (IDT) [8]. The RSAW resonant frequency and wavelength are given by the IDT finger spacing and the respective sound velocity. The resulting SAW amplitude depends on the IDT layout, the applied RF power, and the substrate type, but rarely exceeds the low nanometer range. The RSAW amplitude differences of the 2 modes in the 2 orthogonal crystal directions can be controlled by the IDT layout [9].

The RSAW displacement normal to the surface leads to the most effective excitation of longitudinal acoustic waves

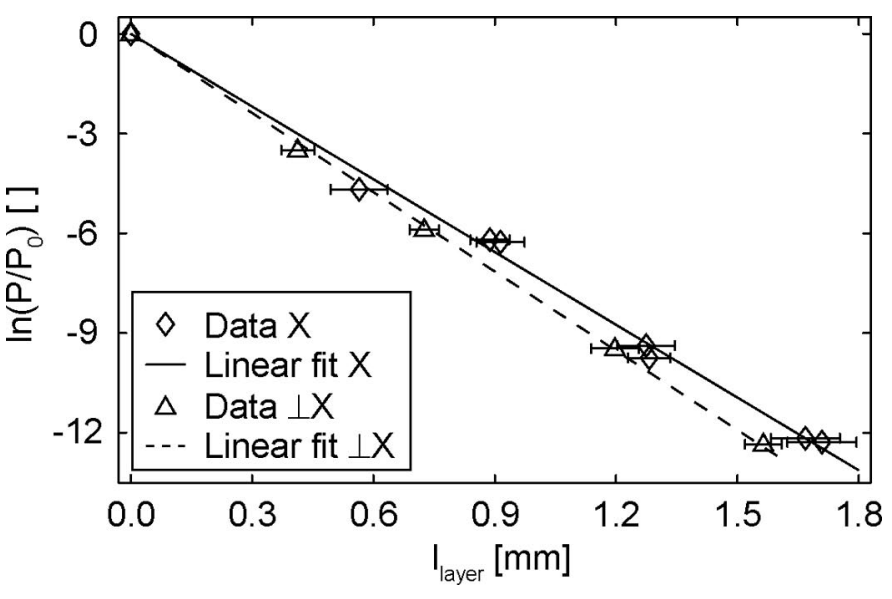

Fig. 2. Force model measurement: The ratio of transmitted radio frequency $(\mathrm{RF})$ power $P / P_{0}$ decays exponentially with respect to the length of the liquid layer layer and so does the RSAW intensity. For direction $X$ and direction $\perp X$, the slopes must differ because of the anisotropic substrate properties. Yet, the correction factors for (2) remain equal.

in the fluid. These acoustic waves propagate within the fluid into the direction of the Rayleigh angle

$$
\Theta_{R}=\arcsin \left(v_{\mathrm{H}_{2} \mathrm{O}} / v_{\mathrm{RSAW}}\right)
$$

which is determined by the ratio of the velocity of the RSAW, $v$ RSAW, and the sound velocity of water $v_{\mathrm{H}_{2} \mathrm{O}}$. The irradiation of sound waves leads to an attenuation of the surface acoustic wave, which then decays with the distance that it has propagated under the fluid. For a sketch of such a leaky RSAW see Fig. 1. According to the perturbation theory by Dransfeld and Salzmann [10] this attenuation is exponential.

We tested the exponential attenuation of the RSAW in sensory experiments by measuring the ratio of the RF power applied to an IDT and of the RF power that is still transmitted after the RSAW has traveled along a path of given length under a layer of water for different path lengths. Fig. 2 confirms this exponential attenuation. According to [10], the characteristic length of attenuation lRSAW of the RSAW-intensity is given by a semi-empirical formula reading

$$
l_{\mathrm{RSAW}}=c \frac{\rho_{\mathrm{RSAW}} v_{\mathrm{RSAW}}}{\rho_{\mathrm{H}_{2} \mathrm{O}} \mathrm{H}_{2} \mathrm{O}} \lambda_{\mathrm{RSAW}} .
$$

It relates the attenuation length to the wavelength $\lambda_{\text {RSAW }}$ of the RSAW, the densities $\rho_{\text {RSAW, } \mathrm{H}_{2} \mathrm{O}}$, and velocities $v_{\mathrm{RSAW}, \mathrm{H}_{2} \mathrm{O}}$ of the substrate and the fluid, respectively. In this formula, $c \approx 0.45$ denotes an empirical factor that assumes the same value for both RSAW propagation directions. This is understood by the fact that in both directions the same mode of the RSAW is excited, hence leading to the exact same interaction with the fluid. However, caused by the different RSAW velocities, the absolute values of the decay constants turn out to 
be different for both directions. For a typical frequency of $f=150 \mathrm{MHz}$ as used in our experiments, the attenuation lengths are $l_{\mathrm{RSAW}}=137 \mu \mathrm{m}$ for the $\mathrm{X}$ direction, and $l_{\mathrm{RSAW}}=126 \mu \mathrm{m}$ for the direction perpendicular to $\mathrm{X}$.

\section{MODELING}

Assuming that the propagation of the leaky RSAW on the substrate is known, one must solve the full nonlinear set of hydrodynamic equations consisting of the Navier-Stokes equations, the continuity equation, and an equation of state for a compressible fluid driven by the time-dependent boundary condition that results from the RSAW. This coupled set of equations has to be solved in a 3-D domain that is determined by the outer geometry of the fluid. Moreover, appropriate inner boundary conditions on the surfaces of the objects immersed in the fluid must be taken into account. These objects themselves move under the action of forces and torques that are exerted from the fluid. This motion then acts back on the fluid via the boundary conditions that the particles impose on the fluid.

Here, we considerably simplify this problem by ignoring those back reactions, treating the particles as entirely passive tracers. In particular, we will completely ignore the modification of the fluid motion by the presence of the particles. However, the remaining fluidic problem is too complicated to be solved numerically.

To further simplify the problem, we also make use of the large time-scale separation between the fast acoustic processes and the slow modes of the fluid motion. The link between these 2 regimes will be provided by the theory of acoustic streaming [11], which allows one to determine the slow motion of the fluid as the solution of a Stokes equation with an effective force term resulting from the fast acoustic velocity field.

\section{A. Raytracing}

The generation and propagation of the acoustic waves within the fluid is governed by the linearized hydrodynamic equations. In contrast to standard acoustic problems [12], it is important to keep the dissipative effects resulting from viscosity and heat conduction. The resulting attenuation of the acoustic waves is crucial for the effect of acoustic streaming. This linearized acoustic problem still poses severe numerical difficulties. It has been solved only for a 2-D model by means of a finite element method (FEM) [13]. Unfortunately, boundary element methods cannot be applied here because the necessary Green functions are unknown for the linearized hydrodynamic problem in a confined geometry and in the presence of dissipation.

A substantial simplification results from the fact that the acoustic wavelength is short compared with the typical extension of the considered fluid volume such that a ray approximation becomes appropriate [14]. The part of the substrate that is covered by the fluid is partitioned into nonoverlapping squares of side length $d_{r s}$. Each of these segments is supposed to emanate a ray that lies in the plane spanned by the vector pointing into the propagation direction of the RSAW and the normal vector on the substrate and encloses the Rayleigh angle with the substrate normal; compare (1) and Fig. 1. In leading approximation, the slow motion of the fluid can be ignored such that the rays extend along straight lines until they meet one of the possible boundaries between the water and either glass, the substrate, or air, where the ray undergoes a total reflection. Each ray propagating into the direction characterized by the unit vector $\hat{\mathbf{k}}$ carries the acoustic fluid velocity

$$
\mathbf{u}_{\mathbf{1}}(\mathbf{x}, t)=u_{0} \hat{\mathbf{k}} e^{-l(\mathbf{x}) / L} \sin (\omega t-\mathbf{k} \cdot \mathbf{x}+\varphi),
$$

where $u_{0}$ denotes the amplitude, $l(\mathbf{x})$ the path length along the ray from its origin at the substrate up to the point $\mathbf{x}$, $\omega=2 \pi \cdot f$ the angular frequency imposed by the RSAW, $\mathbf{k}$ the wave vector of the acoustic wave, and $L$ the decay length. In water $L$ assumes the approximate form [15]

$$
L_{\mathrm{H}_{2} \mathrm{O}}=\frac{8 \pi^{2}}{\left[\alpha\left(T-T_{0}\right)^{2}+\beta\right] \omega^{2}} .
$$

The temperature $T$ is measured in ${ }^{\circ} \mathrm{C}, T_{0}=38^{\circ} \mathrm{C}$ is a reference temperature and $\alpha=4.8 \cdot 10^{-17} K^{2} \mathrm{~m}^{-1} \mathrm{~s}^{-2}$ and $\beta=3 \cdot 10^{-14} \mathrm{~m}^{-1} \mathrm{~s}^{-2}$ are empirical constants. For $T=$ $20^{\circ} \mathrm{C}$ and $f=150 \mathrm{MHz}$ the decay length results as $L_{\mathrm{H}_{2} \mathrm{O}}$ $=1.95 \mathrm{~mm}$. Hagen proposed a length that is about $7 \%$ smaller [16].

The amplitude $u_{0}$ is maximal at the contact line where the RSAW enters the substrate area covered by water. We compute the free surface RSAW-intensity distribution by the angular spectrum of waves method (ASoW) [17]. Hereby, the relevant inputs are the IDT design and the substrate properties [18]. Below the liquid, we assume an exponential decay [10] with characteristic decay length given by (2).

According to the theory of acoustic streaming [11], the acoustic velocity field (3) leads to a body force $\mathbf{f}$ that acts on long-time scales on the fluid. This gives rise to a slow velocity field $\mathbf{v}$ that satisfies a stationary Stokes equation of the form

$$
-\nabla p+\eta \Delta \mathbf{v}+\mathbf{f}=0
$$

where $p$ denotes the pressure and $\eta$ the shear viscosity of the fluid. On the long-time scale, fluids like water can be considered as incompressible, whence

$$
\nabla \cdot \mathbf{v}=0
$$

The slow velocity component $\mathbf{v}$ vanishes at all solid boundaries, including the substrate on which the RSAW 
propagates. We will assume that the pressure field and the streaming of the fluid only lead to negligible deformations of the fluid volume at its free boundaries relative to the equilibrium shape of the fluid. This assumption is well justified, because the SAW power applied while measuring the streamline profiles is far $(-20 \mathrm{~dB})$ lower than the one required for droplet actuation, where we indeed observe boundary deformations. Hence, the boundary is imposed and assumed to have its equilibrium shape. Hence, the boundary is imposed by the equilibrium shape of the fluid. Through this boundary the fluid does not flow, i.e.,

$$
v_{i} N_{i}=0
$$

and a perfect slip condition holds, which reads [19]

$$
T_{i}^{\alpha}\left(\frac{\partial v_{i}}{\partial x_{j}}+\frac{\partial v_{j}}{\partial x_{i}}\right) N_{j}=0 \quad \text { for all } \alpha
$$

where the fields $\mathbf{N}$ and $\mathbf{T}^{\alpha}, \alpha=1,2$ are defined on the free surface and denote the fields of normal and tangential unit vectors, respectively. In the case of strong acoustic driving leading to shape deformations, the boundary conditions have to be supplemented by the balance of the normal stress at the boundary, which then must be determined self-consistently [2].

\section{B. Acoustic Streaming}

The force field $\mathbf{f}$ that drives the slow velocity field $\mathbf{v}$ can be obtained within a perturbative treatment of the Navier-Stokes equations. The unperturbed solution is given by the acoustic velocity and pressure fields resulting from the linearized Navier-Stokes equations. Taking into account the nonlinear advective term one obtains in leading order for the force within a perturbation theory in terms of the unperturbed, acoustic velocity field $\mathbf{u}_{1}[11]$ :

$$
\mathbf{f}=-\rho_{0}\left\langle\left(\mathbf{u}_{1} \cdot \nabla\right) \mathbf{u}_{1}+\mathbf{u}_{1}\left(\nabla \cdot \mathbf{u}_{1}\right)\right\rangle
$$

A detailed description of this theory can be found in [11]. Here, $\mathbf{u}_{1}$ is the acoustic velocity field, $\rho_{0}$ denotes the density of the fluid under thermal equilibrium conditions and the angular brackets represent time averages of a time-dependent field $F(\mathbf{x}, t)$ over one period of the acoustic wave, i.e., $\langle F\rangle=\omega /(2 \pi) \int_{0}^{2 \pi / \omega} d t F(\mathbf{x}, t)$. This average eliminates the fast time-dependence of the acoustic wave. The spatial dependence is not involved in this average such that the resulting force density in general still is a function of space.

The acoustic field belonging to a single ray is given by (3). With (9), its contribution to the streaming force is readily calculated to yield

$$
\mathbf{f}=\frac{u_{0}^{2} \rho_{0}}{L} e^{-2 l(\mathbf{x}) / L} \hat{\mathbf{k}}
$$

This force was computed at a distance $d_{i t}$ along each ray that emanates from a square of side length $d_{r s}$, partitioning the fluid-covered substrate. The forces resulting from all rays crossing a rectangular volume element of side lengths $d_{x}, d_{y}, d_{z}$ were summed to yield a single force for each point of a 3-D rectangular grid covering the total fluid volume. In our calculations, the side lengths were of the order of $15 \mu \mathrm{m}$. By this procedure, possibly existing phase relationsships between the waves belonging to different rays were ignored. In particular, close to the boundaries the spatial patterns resulting from the interference between incoming and reflecting waves are suppressed by this approximation. In most cases, these interference patterns are characterized by a short length scale of the order of the wavelength of the acoustic wave.

We conclude that the theory of acoustic streaming predicts the force entering the Stokes equation (5) to be proportional to the sound intensity and hence, via the RSAW intensity, to the RF power [20]. Because of the linearity of the Stokes equation this implies that the slow fluid velocity is directly proportional to the RF power applied to the IDT.

To confirm this prediction, the velocity field in a water layer sandwiched between 2 parallel glass plates was monitored by a particle-tracking technique [21]. A sketch of the experiment is displayed in Fig. 3. The plates rest on a piezoelectric substrate on which an RSAW propagates parallel to the plates. The velocity was measured at different plate positions for various RF powers applied to the IDT causing the RSAW. Histograms of ratios $q_{u}=$ $u(x, y) / u_{0}(x, y)$ of the velocities at a power $P$ and respective velocities at a reference power $P_{0}$ were compiled from the measurements. In Fig. 4, the most probable values estimated from the histograms are plotted as a function of the ratio $q P$ of corresponding $\mathrm{RF}$ powers. This result is in good agreement with the proportionality of the fluid velocity and the applied RF power predicted by the theory of acoustic streaming.

An absolute agreement of the numerical and experimental velocity fields could not be achieved most likely because the RSAW amplitude has not been known precisely enough. We corrected for this mismatch by fitting the velocity field to the experimentally determined value at the single point where both fields attain their maximal values. In this way, we always obtained quantitative consistency with the experiments described below in more detail.

\section{Particle Advection}

Once the force field $\mathbf{f}$ is known on a sufficiently fine grid, the Stokes equation (5) can be solved numerically. In the resulting flow pattern, small particles that are suspended in the fluid are advected, i.e., they follow the streamlines of the velocity field v. Consequently, the particle trajectories are solutions of the equation

$$
\frac{d \mathbf{x}}{d t}=\mathbf{v}(\mathbf{x})
$$




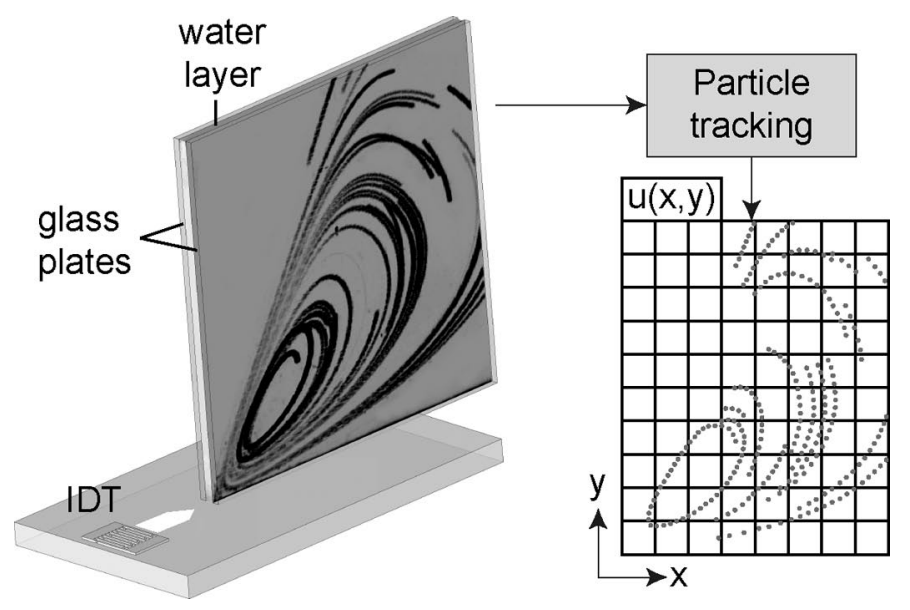

Fig. 3. An IDT creates a flow pattern in a water layer confined within 2 parallel glass plates (RSAW path sketched by an arrow). In this setup, the relationship between the slow fluid velocity $u(x, y)$ (derived by particle tracking) and the applied RF power is investigated.

\section{Implementation}

Several software components are involved in the RSAWRaytracing simulation (see Fig. 5). At first, the shape of the water volume is determined by the Surface Evolver [22] and exported for Mathematica (Wolfram Research, Inc., Champaign, IL) and Comsol Multiphysics (Comsol, Inc., Stockholm, Sweden). In Mathematica, a part of the model file is selected and transferred to the RSAW-Raytracing application. Furthermore, it receives the RSAW-intensity distributions for all IDTs in use, acquired by the ASoW method in Mathematica. The resulting acoustic streaming force field can be visualized in Mathematica and is imported as linearly interpolated function into Comsol Multiphysics. Here, the Stokes equation (5) is solved using FEM with approximately 30,000 tetrahedral elements with $45 \mu \mathrm{m}$ edge lengths. The used basis functions are second-order Lagrange elements for the velocity field and first-order Lagrange elements for the pressure. The streamline plots are generated for the comparison to the recorded fluorescent particle trajectories.

\section{Comparison of Experiments And Simulation}

Our templates for multi-purpose microfluidic studies possess 12 quadratic electrodes for flexible contacting with cantileverlike pins. The $100 \mathrm{~nm}$ gold structures like IDTs and fluid reservoir floors (for contrast enhancement in fluorescent microscopy) are covered by a $150 \mathrm{~nm} \mathrm{SiO}$ protection layer. In areas surrounding the desired reservoir, the hydrophilic SiO surface is turned hydrophobic by OTS (octadecyl trichlore silane). These chips are inserted into a cooled sample holder to reduce evaporation.

To verify the quality of the raytracing approach, we carried out experiments in various geometries, 2 of which are presented here. In the first case of Fig. 6(a), we cover the $0.33 \mu \mathrm{l}$ water droplet with a glass slide at distance 300 $\pm 10 \mu \mathrm{m}$ so that it takes the shape of a catenoid, which

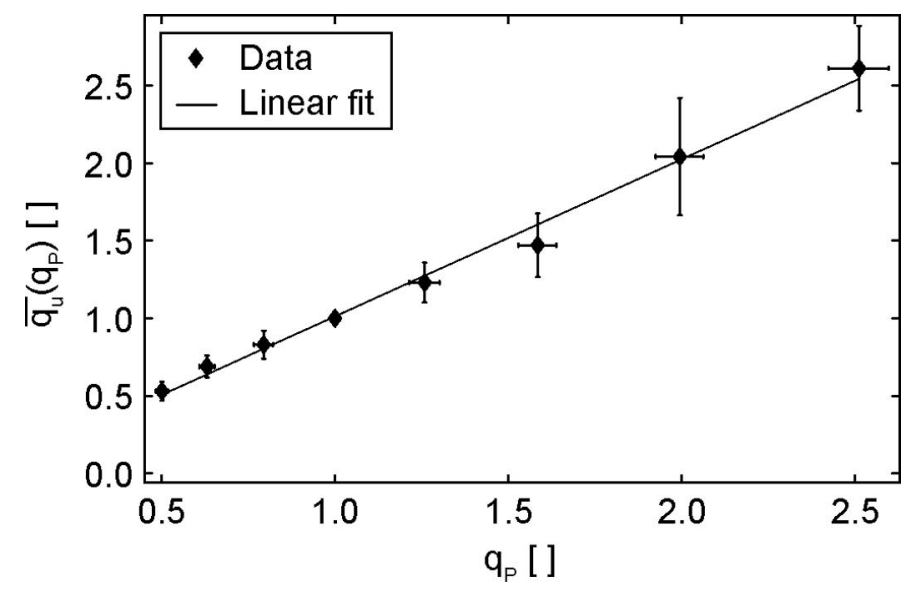

Fig. 4. Force model measurement: The ratio of applied RF powers $q_{p}$ is proportional to the ratio of fluid velocities $\overline{q_{u}}$ with slope 1 . Hence, the fluid velocity is proportional to the local sound intensity in water.

is a cylinder with a concave mantel. Now, a tapered IDT [23] allows the excitation of a narrow horizontal RSAW path (full-width half-maximum $115 \pm 5 \mu \mathrm{m}$ ) whose impact position can be adjusted by the RF frequency along the catenoid's right half-circle. The second example of Fig. 6(b) deals with a typical microfluidic circuit, defined on the substrate by surface wettability modulation. The nontrivial, self consistently resulting shape of a small $(0.8 \mu \mathrm{l})$ amount of water, spotted onto the circuit, is numerically calculated by the Surface Evolver. In both systems, the flow of fluorescent particles (radius $2.3 \mu \mathrm{m}$ ) was recorded to obtain trajectory images by superimposing the movie frames.

In Fig. 7(a)-(c), the RSAW path position is indicated by an arrow being shifted from the center in part (a) to an intermediate position in (b) and to the outer region in (c) of the catenoid. Accordingly, Fig. 7(a) shows a symmetric flow pattern. By contrast, we observed a spreading flow pattern in Fig. 7(b) caused by the meniscus deflecting a fraction of the high-intensity sound waves where

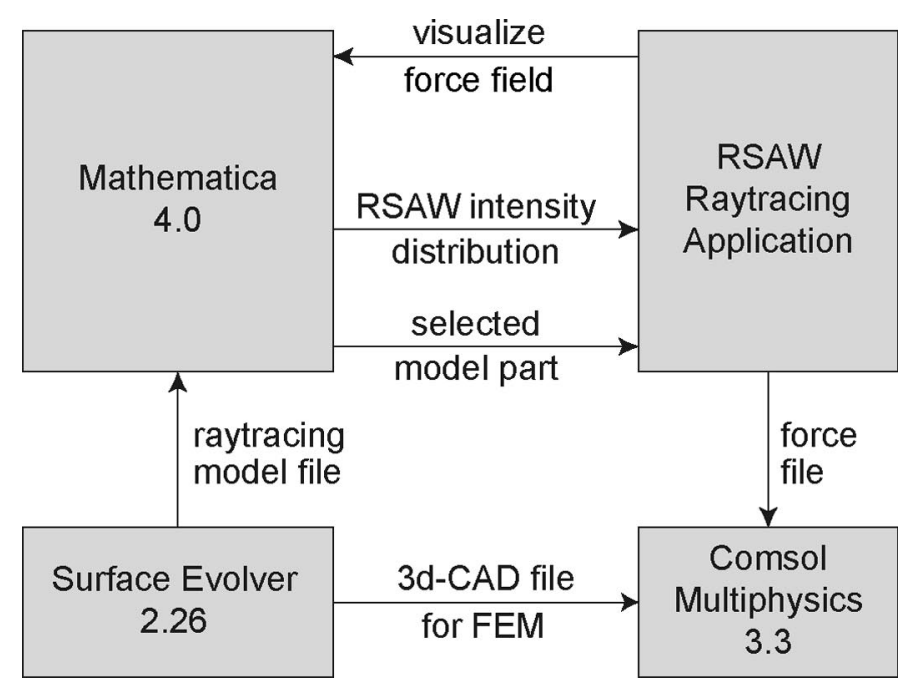

Fig. 5. Software components: The simulation of RSAW microfluidics is handled by multiple applications, as described in the text. 

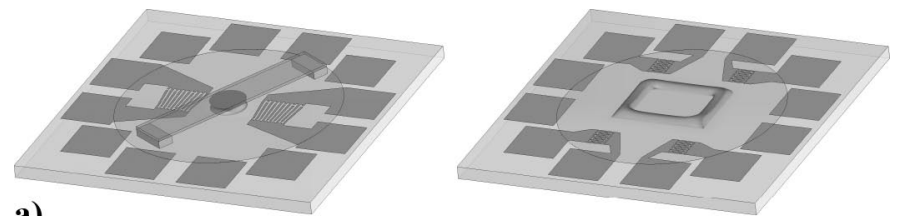

a)

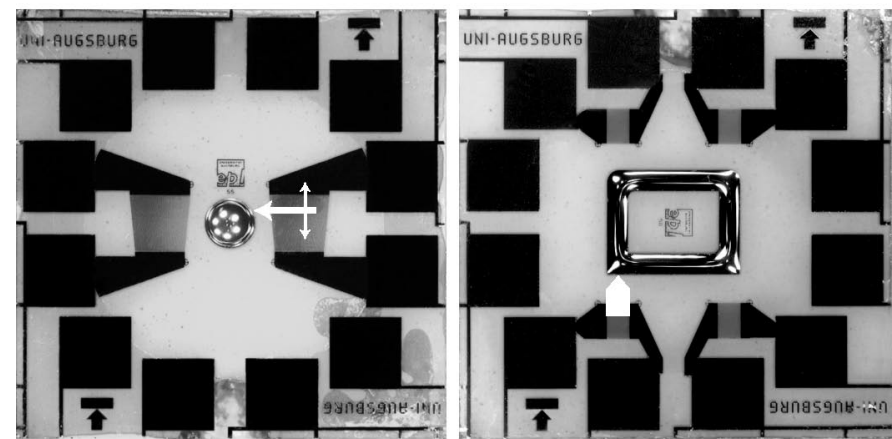

b)

Fig. 6. RSAW microfluidics chips: The top row depicts the schematic 3-D layout of the chip, while the bottom row contains top views of the samples with sketched RSAW paths. (a) A water reservoir, covered by a thin glass slide (omitted in the photgraph for clarity) can be examined by local actuation with narrow RSAW beams excited and shifted by a tapered IDT. (b) A free-surface microfludic circuit is driven by a wide fixed RSAW beam of a normal IDT injected in a corner.
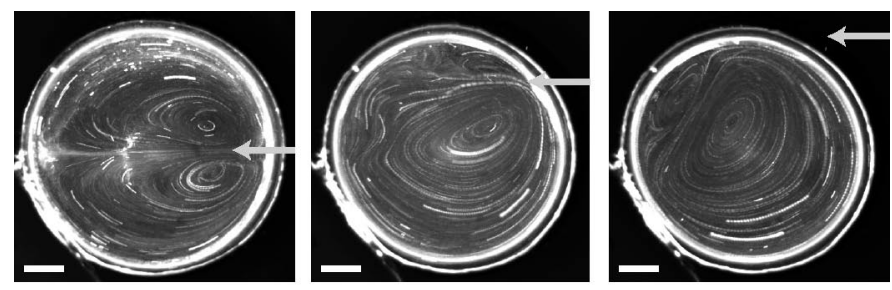

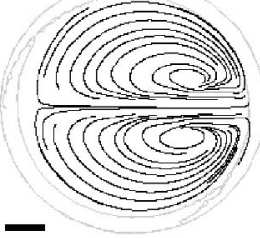

a)

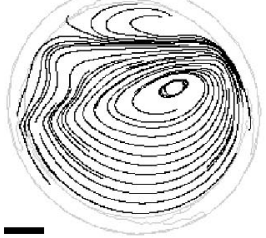

b)

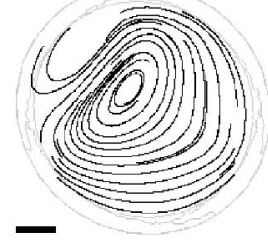

c)
Fig. 7. Experiments in a catenoid: A narrow RSAW path is applied at a specific position (marked by the arrow): (a) center, (b) intermediate, and (c) outer part. The upper row images depict particle trajectories for different RSAW beams, while the bottom row contains the coincident streamlines of the RSAW-Raytracing simulation (scale bar $200 \mu \mathrm{m}$ ). The temperature was set to $T=20^{\circ} \mathrm{C}$ in this experiment.

they couple into the water. In the last case of Fig. 7(c), all sound waves are reflected at the meniscus, leading to a flow pattern significantly tilted against the RSAW direction. All 3 examples are reproduced excellently by the RSAW-Raytracing simulation regarding the vortex positions and streamlines.

Fig. 8(a) displays the force field $\mathbf{f}$ that results from a wide RSAW path (fwhm $490 \pm 10 \mu \mathrm{m}$ ) in a knee-shaped fluid with a free surface. The involved structure of the force field is a consequence of successive reflections at the bent free surfaces. In Fig. 8(b), the streaming pattern re-

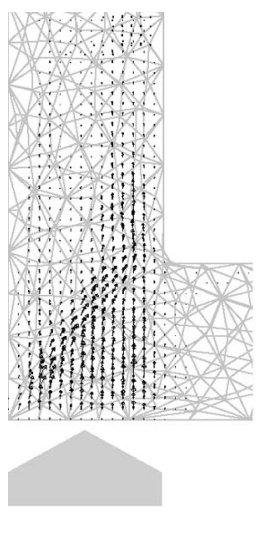

a)

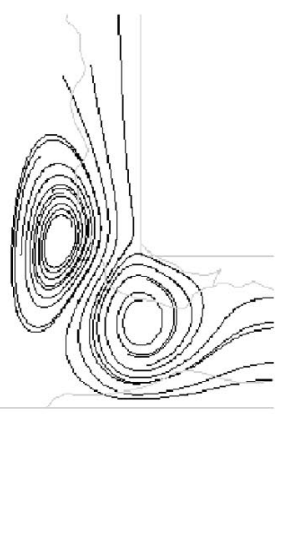

b)

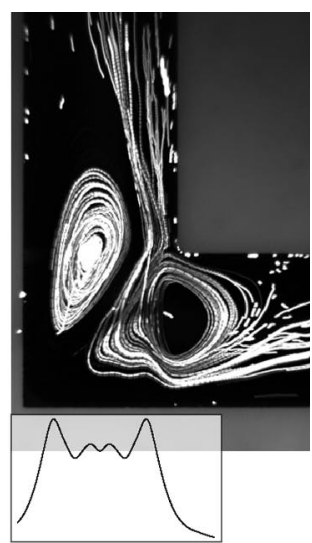

c)
Fig. 8. Results in a free surface corner: (a) The bent free surface creates a complex force field arising from a wide RSAW path coupled into the corner (3-D resolution coarsened by a factor 20 for clarity). (b) The tilt of the simulated flow pattern against the RSAW direction and both vortices are reproduced in detail by the experiments in (c). In the near-field of the strongest radiation sources, the particles experience further forces (scale bar $200 \mu \mathrm{m}$ ). Inset: RSAW intensity calculated by the ASoW method. The temperature of this experiment was $T=15.5^{\circ} \mathrm{C}$.

sulting from the solution of the Stokes equation (5) is compared with the trajectories of small beads in an experiment with the same geometry and RSAW actuation in Fig. 8(c). Apart from deviations in the region of the strongest RSAW intensity, the agreement is excellent. A possible reason for the observed deviations is the finite extension of the particles in combination with possibly high pressure gradients.

\section{CONCLUSION}

By applying a raytracing algorithm we were able to determine the RSAW-induced flow patterns and the transport of small particles in microfluidic sytems of arbitrary shape. The acoustic streaming force model contains only a single scaling parameter characterizing the maximum fluid velocity. The developed software package is able to analyze a system comprehensively from the RSAW excitation to the observed microfluidics in less than $2 \mathrm{~h}$. At present, we employ this software routinely to optimize mixing and sorting tasks. Moreover, our concept can be transferred to acoustic streaming systems with different sound sources, like bulk acoustic waves.

\section{ACKNOWLEDGMENTS}

Valuable discussions with Werner Ruile, Daniel Köster, and Michael Schindler are gratefully acknowledged. The authors thank A. Spörhase, S. Lieber, and A. Hupfer for their technical assistance.

\section{REFERENCES}

[1] K. Sritharan, C. J. Strobl, M. F. Schneider, Z. Guttenberg, and A. Wixforth, "Acoustic mixing at low Reynold's numbers," Appl. Phys. Lett., vol. 88, no. 5, art. no. 054102, 2006. 
[2] M. Schindler, P. Talkner, and P. Hänggi, "Computing stationary free surface shapes in microfluidics," J. Fluid Mech., vol. 18, no. 10, art. no. 103303, 2006.

[3] Z. Guttenberg, H. Mueller, H. Habermueller, A. Geisbauer, J. Pipper, J. Felbel, M. Kielpinski, J. Scriba, and A. Wixforth, "Planar chip device for PCR and hybridization with surface acoustic wave pump," Lab Chip, vol. 5, no. 3, pp. 308-317, 2005.

[4] A. Rathgeber, M. Wassermeier, and A. Wixforth, "Acoustic 'distributed source' mixing of smallest fluid volumes," J. ASTM Int., vol. 2, no. 6 , p. $6,2005$.

[5] A. Wixforth, C. J. Strobl, C. Gauer, A. Toegl, J. Scriba, and Z. Guttenberg, "Acoustic manipulation of small droplets," Anal. Bioanal. Chem., vol. 379, no. 7-8, pp. 982-991, 2004.

[6] T. M. Squires and S. R. Quake, "Microfluidics: Fluid physics at the nanoliter scale," Rev. Mod. Phys., vol. 77, pp. 977-1026, 2005.

[7] L. J. W. S. Rayleigh, "On waves propagating along the planar surface of an elastic solid," Proc. Lond. Math. Soc., vol. 17, no. 3, pp. $4-11,1885$.

[8] R. M. White and F. W. Voltmer, "Direct piezoelectric coupling to surface elastic waves," Appl. Phys. Lett., vol. 7, no. 12, pp. 314-316, 1965.

[9] G. W. Farnell, "Elastic surface waves," in Surface Wave Filters, H. Matthews, Ed. New York: Wiley, 1977, pp. 109-166.

[10] K. Dransfeld and E. Salzman, "Excitation, detection, and attenuation of high-frequency elastic surface waves," in Physical Acoustics, vol. 7, W. P. Mason and R. N. Thurston, Eds. New York: Academic Press, 1970, pp. 219-272.

[11] W. L. M. Nyborg, "Acoustic streaming," in Physical Acoustics, vol. 2B, W. P. Mason, Ed. New York: Academic Press, 1965, pp. 256331.

[12] P. M. Morse and K. U. Ingard, Theoretical Acoustics. New York: McGraw-Hill, 1968.

[13] D. Köster, "Numerical simulation of acoustic streaming on surface acoustic wave-driven biochips," SIAM J. Sci. Comput., vol. 29, no. 6, pp. 2352-2380, 2007.

[14] L. D. Landau and E. M. Lifshitz, Fluid Mechanics. New York: Pergamon, 1959.

[15] R. F. W. Coates, Underwater Acoustic Systems. New York: Wiley, 1989.

[16] R. Hagen, R. Behrends, and U. Kaatze, "Acoustical properties of aqueous solutions of urea: Reference data for the ultrasonic spectrometry of liquids," J. Chem. Eng. Data, vol. 49, no. 4, pp. 988-991, 2004.

[17] G. Visintini, Modellierung von Oberflächenwellenfiltern im Wellenvektorraum. Düsseldorf: VDI, 1990. (in German)

[18] A. J. Slobodnik, E. D. Conway, and R. T. Delmonico, Microwave Acoustics Handbook, 2nd ed. Air Force System Command, U. S. Air Force, 1973.

[19] M. Schindler, P. Talkner, M. Kostur, and P. Hänggi, "Accumulating particles at the boundaries of a laminar flow," Physica A, vol. 385, no. 1 , pp. $46-58,2007$.

[20] S. Datta, Surface Acoustic Wave Devices. Englewood Cliffs, NJ: Prentice Hall, 1986.

[21] Z. Guttenberg, A. Rathgeber, S. Keller, J. O. Rädler, A. Wixforth, M. Kostur, M. Schindler, and P. Talkner, "Flow profiling of a surface-acoustic-wave nanopump," Phys. Rev. E Stat. Nonlin. Soft Matter Phys., vol. 70, no. 5, art. no. 056311, 2004.

[22] K. Brakke, "The surface evolver," Experimental Mathematics, vol. 1, no. 2, pp. 141-165, 1992.

[23] M. Streibl, A. Wixforth, J. P. Kotthaus, A. O. Govorov, C. Kadow, and A. C. Gossard, "Imaging of acoustic charge transport in semiconductor heterostructures by surface acoustic waves," Appl. Phys. Lett., vol. 75, no. 26, pp. 4139-4141, 1999.

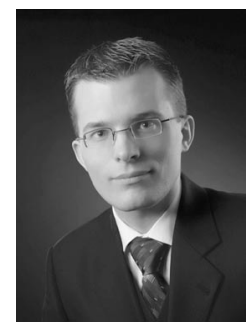

Thomas Frommelt was born in Augsburg, Germany, in 1978. He received the Dipl. degree in physics in 2003 from the University of Augsburg for his thesis on plasma enhanced deposition of thin films under atmospheric conditions. In 2004, he joined the microfluidics group of Prof. Wixforth and focused on the simulation of surface acoustic wave driven microfluidics for application. The resulting toolset was used for mixing optimization by chaotic advection in theory and experiment. In 2007, he received his Dr. rer. nat. degree from the University of Augsburg for this thesis work and was awarded several prizes. Currently, he is working as a project manager in the modeling department of the SGL Group.

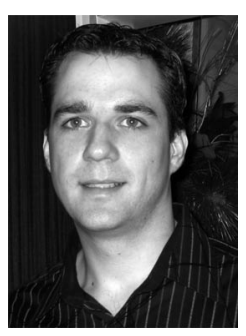

Daniel Gogel was born in Dachau, Germany, in 1982. He has been studying material science at the University of Augsburg since 2003. In 2006, he joined the microfluidics group of Prof. Wixforth and received the B.S. degree for his thesis on driven fluidics in open and closed geometries. Since 2008, he has been working with Prof. Lindner for his master thesis on structuring self-arranged colloidal nano-masks.

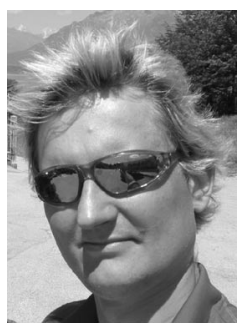

Marcin Kostur currently works as an assistant professor at the Institute of Physics at the University of Silesia in Katowice, Poland. He obtained his Ph.D. degree in the field of stochastic dynamics in 1999. He spent a few years doing postdoctoral work at the Institutes of Physics at Humboldt University in Berlin, Germany; the University of Maine; and the University of Augsburg, Germany. His field of personal research interest and activity concerns transport properties of stochastic systems and their applications to the modeling of molecular motors, Josephson junctions, and microfluidic systems. He has specialized in numerical methods in stochastic dynamics, Fokker-Planck equations, and high-performance parallel computing.

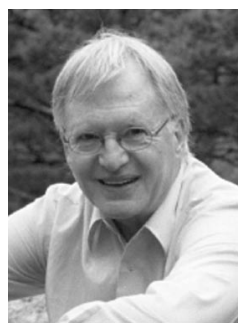

Peter Talkner received the Diploma and Dr. rer. nat. degrees from the Institute of Physics at the University of Stuttgart, Germany, in 1972 and 1979, respectively. In 1984 he obtained the Habilitation in Theoretical Physics degree from the Institute of Physics at the University of Basel, Switzerland. From 1989 until 2002, he was the head of the Section Stochastic Systems of the Paul Scherrer Institute in Villigen, Switzerland. In 2000, he became a professor at the University of Basel. Since 2002, he has worked as a senior scientist in the Institute of Physics at the University of Augsburg, Germany. His research interests include various aspects of nonequilibrium statistical mechanics, such as open quantum systems, thermally activated rates, transport in driven systems as well as time series analysis and microfluidics. In 2008, he was selected by the American Physical Society as an Outstanding Referee.

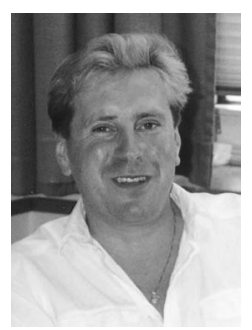

Peter Hänggi received the Diploma and Ph.D, degrees in theoretical physics from the University of Basel, Switzerland, in 1974 and 1977, respectively. He is an elected Fellow of the American Physical Society (APS), the Institute of Physics (IoP), and the American Association for the Advancement of Science (AAAS) (2005) as well as an elected external member of the Max-Planck Institute for Physics of Complex Systems (MPI-PKS) and elected member of the Max Planck Society. He is recipient of the Humboldt Honorary Research Award of the Foundation of Polish Science (2002); the Eminent Scientist Award, RIKEN (2004); the Smoluchowski-Medal of the Jagellonian University of Krakow, Poland (2006); and the Elena Aizen de Moshinsky Professorship, UNAM, Mexico City (2006). Peter Hanggi also has been awarded doctor honoris causa degrees from the University of Silesia, Katowice, Poland; Universitat de Barcelona, Spain; University of Camerino, Italy; the Tartar State University of Humanities and Pedagogical Sciences, Kazan, Russia; the National Academy of Ukraine, Kiev, Ukraine; and Humboldt University in Berlin. 


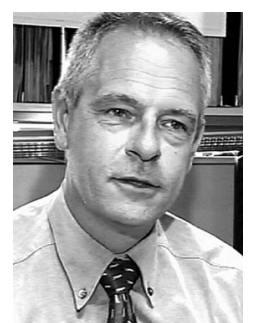

Achim Wixforth is full professor for experimental physics at the University of Augsburg, Germany. There, his research efforts include semiconductor nanophysics and technology, material science, microfluidics, and biophysical topics. In 2000, he founded the Advalytix AG, a spinoff company of the Center for NanoScience (CeNS) of the University of Munich, which has recently been acquired by Olympus Life and Material Science, Europe.

He received his $\mathrm{Ph} . \mathrm{D}$. degree in physics from the University of Hamburg, Germany, in 1987. He spent a postdoctoral leave at the University of California, Santa Barbara, where he collaborated with Professors Gossard, Kroemer, and Petroff as an assistant research engineer. Back in Germany, he received his Habilitation degree from the University of Munich in 1994 where he was senior scientist and lecturer before accepting the chair in Augsburg. He is author and coauthor of more than 170 scientific papers and book chapters and looks back on a long list of invited talks. Among a variety of other awards, he received in 1998 the Walter Schottky Prize for solid-state research, one of the most prestigious scientific awards in Germany, and in 2003 the first BioTrends Award. 